\title{
PLANTAR THROMBOPHLEBITIS: MAGNETIC RESONANCE IMAGING FINDINGS
}

Frederico Celestino Miranda', Renato Duarte Carneiro², Carlos Henrique Longo ${ }^{3}$, Túlio Diniz Fernandes ${ }^{4}$, Laércio Alberto Rosemberg ${ }^{5}$, Marcelo Buarque de Gusmão Funari ${ }^{6}$

\section{ABSTRACT}

Objective: Demonstrate the magnetic resonance imaging (MRI) findings in plantar thrombophlebitis. Methods: Retrospective review of twenty patients with pain in the plantar region of the foot, in which the MRI findings indicated plantar thrombophlebitis. Results: A total of fourteen men and six women, mean age 46.7 years were evaluated. Eight of these patients also underwent Doppler ultrasonography, which confirmed the thrombophlebitis. The magnetic resonance images were evaluated in consensus by two radiologists with experience in musculoskeletal radiology (more than 10 years each), showing perivascular edema in all twenty patients $(100 \%)$ and muscle edema in nineteen of the twenty patients (95\%). All twenty patients had intraluminal intermediate signal intensity on T2-weighted (100\%) and venous ectasia was present in seventeen of the twenty cases (85\%). Collateral veins were visualized in one of the twenty patients $(5 \%)$. All fourteen cases $(100 \%)$, in which intravenous contrast was administered, showed perivenular tissues enhancement and intraluminal filling defect. Venous ectasia, loss of compressibility and no flow on Doppler ultrasound were also observed in all eight cases examined by the method. Conclusion: MRI is a sensitive in the evaluation of plant thrombophlebitis in patients with plantar foot pain.

Keywords - Thrombophlebitis; Magnetic Resonance Imaging; Foot

\section{INTRODUCTION}

Plantar thrombophlebitis (PT) is an entity with few cases reported in the literature. The pathogenesis of this condition remains uncertain, and may be related to multiple causes, such as previous surgery, trauma, immobilization, paraneoplastic conditions, genetic mutations of the blood coagulation cascade, and excessive physical activity ${ }^{(1)}$. It is characterized by intraluminal thrombus and edema/inflammation of perivenular tissue ${ }^{(2)}$. Patients report nonspecific pain in the plantar region, making clinical diagnosis very difficult, with numerous differentials, such as intermetatarsal bursitis, Morton's neuroma, sesamoiditis, plantar fasciitis, tendon pathologies, ganglion cysts, and stress fracture. The aim of this study was to demonstrate the PT findings in magnetic resonance imaging (MRI) in patients with pain in the plantar region.

\section{METHODS}

Between March 2006 and September 2009, 20 magnetic resonance imaging (MRI) exams which showed signs of PT were retrospectively evaluated. The mean age was 46.7 years (22-67 years), with 14 men (mean age 47.5 years, 22-67 years) and six women (mean age 44.8 years, $31-66$ years). There was no predominance of laterality, and the right and left foot were involved in 10 cases each. In 12 patients, thrombophlebitis was located in the forefoot and in the hindfoot in eight (Table 1). All patients reported pain in the region affected by thrombophlebitis, and

1 - Radiologist, Hospital Israelita Albert Einstein, São Paulo, SP, Brazil.

2 - Radiologist, Multimagem Diagnósticos, Goiânia, GO, Brazil.

3 - Doctor; Radiologist, Hospital Israelita Albert Einstein, São Paulo, SP, Brazil.

4 - Doctor; Orthopedist, Department of Orthopedics and Traumatology, School of Medicine, Universidade de São Paulo (USP), São Paulo, SP, Brazil.

5 - Doctor, Radiologist, Radiology Institute, Hospital das Clínicas, School of Medicine, Universidade de São Paulo (InRad/HCFMUSP), and Hospital Israelita Albert Einstein, São Paulo, SP, Brazil.

6 - Doctor, Radiologist, Radiology Institute, Hospital das Clínicas, School of Medicine, Universidade de São Paulo (InRad/HCFMUSP); Manager, Imaging Department, Hospital Israelita Albert Einstein, São Paulo, SP, Brazil.

Study conducted at the Hospital Israelita Albert Einstein, São Paulo, SP, Brazil.

Correspondence: Av. Albert Einstein, 627 - $4^{\circ}$ andar/Bloco D - 05652-900 - Morumbi - São Paulo, SP, Brazil. Email: frederico.miranda@einstein.br Received for publication: 3/28/2012, accepted for publication: 4/12/2012..

The authors declare that there was no conflict of interest in conducting this work 
Table 1 - Patient characteristics.

\begin{tabular}{c|c|c|c|c|c}
\hline Case & Sex & Age & Region/Side & GD & USG \\
\hline 1 & $\mathrm{~F}$ & 48 & Left hindfoot & $\mathrm{N}$ & $\mathrm{Y}$ \\
\hline 2 & $\mathrm{~F}$ & 43 & Right forefoot & $\mathrm{Y}$ & $\mathrm{N}$ \\
\hline 3 & $\mathrm{M}$ & 67 & Right forefoot & $\mathrm{Y}$ & $\mathrm{N}$ \\
\hline 4 & $\mathrm{~F}$ & 31 & Right hindfoot & $\mathrm{N}$ & $\mathrm{Y}$ \\
\hline 5 & $\mathrm{M}$ & 49 & Right forefoot & $\mathrm{Y}$ & $\mathrm{N}$ \\
\hline 6 & $\mathrm{M}$ & 47 & Left hindfoot & $\mathrm{Y}$ & $\mathrm{Y}$ \\
\hline 7 & $\mathrm{M}$ & 31 & Right forefoot & $\mathrm{Y}$ & $\mathrm{N}$ \\
\hline 8 & $\mathrm{M}$ & 43 & Left hindfoot & $\mathrm{Y}$ & $\mathrm{Y}$ \\
\hline 9 & $\mathrm{~F}$ & 40 & Right forefoot & $\mathrm{Y}$ & $\mathrm{N}$ \\
\hline 10 & $\mathrm{M}$ & 52 & Right forefoot & $\mathrm{Y}$ & $\mathrm{N}$ \\
\hline 11 & $\mathrm{~F}$ & 66 & Left hindfoot & $\mathrm{N}$ & $\mathrm{Y}$ \\
\hline 12 & $\mathrm{M}$ & 56 & Right forefoot & $\mathrm{Y}$ & $\mathrm{N}$ \\
\hline 13 & $\mathrm{M}$ & 50 & Left hindfoot & $\mathrm{Y}$ & $\mathrm{Y}$ \\
\hline 14 & $\mathrm{M}$ & 45 & Left hindfoot & $\mathrm{N}$ & $\mathrm{N}$ \\
\hline 15 & $\mathrm{~F}$ & 41 & Left forefoot & $\mathrm{Y}$ & $\mathrm{N}$ \\
\hline 16 & $\mathrm{M}$ & 22 & Left forefoot & $\mathrm{Y}$ & $\mathrm{N}$ \\
\hline 17 & $\mathrm{M}$ & 46 & Left forefoot & $\mathrm{Y}$ & $\mathrm{N}$ \\
\hline 18 & $\mathrm{M}$ & 65 & Right forefoot & $\mathrm{Y}$ & $\mathrm{N}$ \\
\hline 19 & $\mathrm{M}$ & 60 & Left forefoot & $\mathrm{N}$ & $\mathrm{Y}$ \\
\hline 20 & $\mathrm{M}$ & 32 & Right hindfoot & $\mathrm{N}$ & $\mathrm{Y}$ \\
\hline
\end{tabular}

GD: Gadolinium (venous contrast);

USG: Ultrasound;

Y:Yes;

$\mathrm{N}:$ No.

eight of them also underwent Doppler ultrasonography, directed to the area of pain, with the confirmation of plantar thrombophlebitis in all cases. One of the patients also had deep venous thrombosis of the ipsilateral leg and another patient had venous thrombosis of the left subclavian vein, one month after the PT, both confirmed with Doppler ultrasonography.

The institutional board approved this study and informed consent was waived.

The MRIs were performed on a 1.5 Tesla magnet (Signa Excite, GE Medical Systems, Milwaukee, WI) using dedicated surface coils. In forefoot exams, axial T2WI fast spin-echo (FSE) (2000-4000/40-70), axial T1WI axial FSE (300-600/9-15), coronal T1WI FSE (400-600/9-15), coronal T2WI FSE with fat suppression (2000-4000/40-70), sagittal T1WI FSE (400-600/9-15), axial post-contrast T1WI FSE with fat suppression (300-600/9-15), and coronal post-contrast T1WI FSE with fat suppression (300-600/9-15) sequences were used. In examinations of the ankle, axial T2WI FSE with fat suppression (3750-5750/40-70), axial T1WI FSE (600-800/9-15), sagittal T1WI FSE (400-600/915), coronal T2WI FSE (2500-4500/40-70), sagittal T2WI FSE with fat suppression (3250-5250/40-70), sagittal T1WI FSE (400-600/9-15) and proton densityWI (1500-3500/30-60) sequences were used.
All MRI exams were performed in a $12-14 \mathrm{~cm}$ field of view (FOV), with a slice thickness of $3.0 \mathrm{~mm}$ and an intersection gap of $1.0 \mathrm{~mm}$, in a $256 \times 224$ matrix. Gadopentetate dimeglumine (Magnevist ${ }^{\circledR}$, Berlex Laboratories, Wayne, NJ) was used as a contrast agent intravenously at a dose of $0.1 \mathrm{mmol} / \mathrm{kg}$ bodyweight in 14 cases. In eight cases, ultrasound examination (USG) was performed after PT diagnosis by MRI using the HDI 5000 (Philips, Washington, USA).

In all cases the patients complained of localized plantar pain. All patients were referred for MRI and clinical suspicions included Morton's neuroma, intermetatarsal bursitis, sesamoiditis, stress fractures, and plantar fasciitis. None had clinical suspicion of plantar thrombophlebitis.

All MRI scans were retrospectively reviewed in consensus by two radiologists with more than 10 years of experience in musculoskeletal radiology. The reviewers had access only to the patients' symptoms, which in all 20 cases was pain in the plantar region. As imaging findings, the following were considered: a) prior to injection of contrast: perivascular edema, muscle edema, characteristics of the intraluminal signal on T2WI and T1WI, venous ectasia and collateral veins, b) after injection of intravenous contrast: perivascular enhancement and failure of intraluminal filling. In addition, the location of thrombophlebitis was evaluated.

Ultrasound examination was performed by a radiologist with over 10 years of experience in the evaluation of the musculoskeletal system and access to the patient's symptoms and the MRI report, indicating PT. Vessel distension, loss of compressibility and absence of doppler flow were considered as imaging findings.

\section{RESULTS}

Imaging findings on magnetic resonance imaging:

\section{Perivascular edema}

All 20 patients (100\%) showed hyperintense signal on T2 (with fat suppression) in the perivascular tissue (Figure 1).

\section{Muscle edema}

Nineteen of 20 patients ( $95 \%$ ) had muscle edema in the region of the vessels involved. 


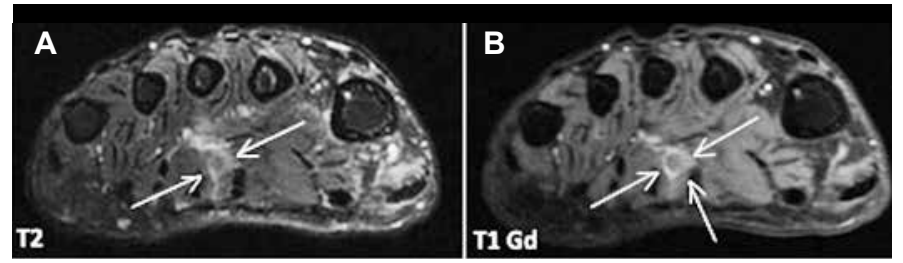

Figure 1 - Female patient, 40 years. Coronal T2WI FSE with fat saturation (A) - Edema in the myoadipose planes, around the plantar metatarsal vein between metatarsals II and III of the right forefoot. Coronal T1WI FSE with fat saturation and contrast (B) - Venular distension with intraluminal filling defect and enhancement of perivascular tissue.

\section{Intraluminal signal}

All patients $(100 \%)$ had intraluminal intermediate signal on T2WI. Seventeen of the 20 patients $(85 \%)$ had intraluminal intermediate signal on T1WI, and three of the 20 patients (15\%) showed intraluminal hyperintense signal on T1WI (Figure 2).
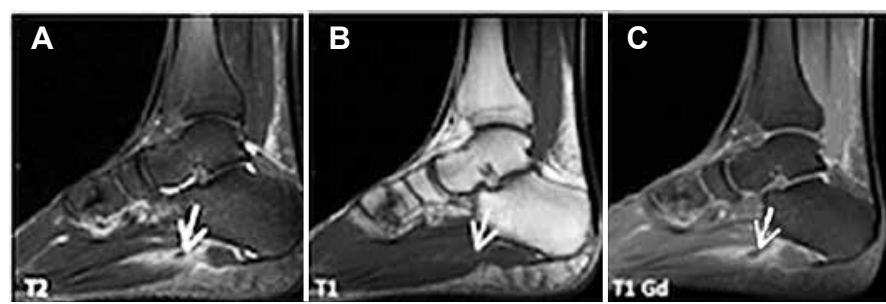

Figure 2 - Male patient, 50 years. Sagittal T2WI FSE with fat saturation and sagittal T1WI FSE (A/B) - Intraluminal intermediate signal in T1WI and T2WI. Sagittal T1WI FSE with fat saturation and contrast (C) - Intraluminal filling defect within the lateral venous system of the hindfoot.

\section{Venous ectasia}

An increase in the diameter of the involved veins was observed in 17 of the 20 cases $(85 \%)$.

\section{Collateral veins}

Collateral veins were noted in one of the 20 cases (5\%), probably representing a chronic case (Figure 3 ).

\section{Perivascular enhancement}

All 14 patients $(100 \%)$ subjected to intravenous contrast showed perivascular enhancement (Figure 4).

\section{Intraluminal filling failure}

All 14 cases (100\%) subjected to intravenous contrast showed a filling defect within the compromised vessel (Figure 5).

\section{Affected veins}

MRI assessed which veins were affected, with the results shown in Table 2. It should be noted that in some cases there was a simultaneous involvement of more than one vein segment.

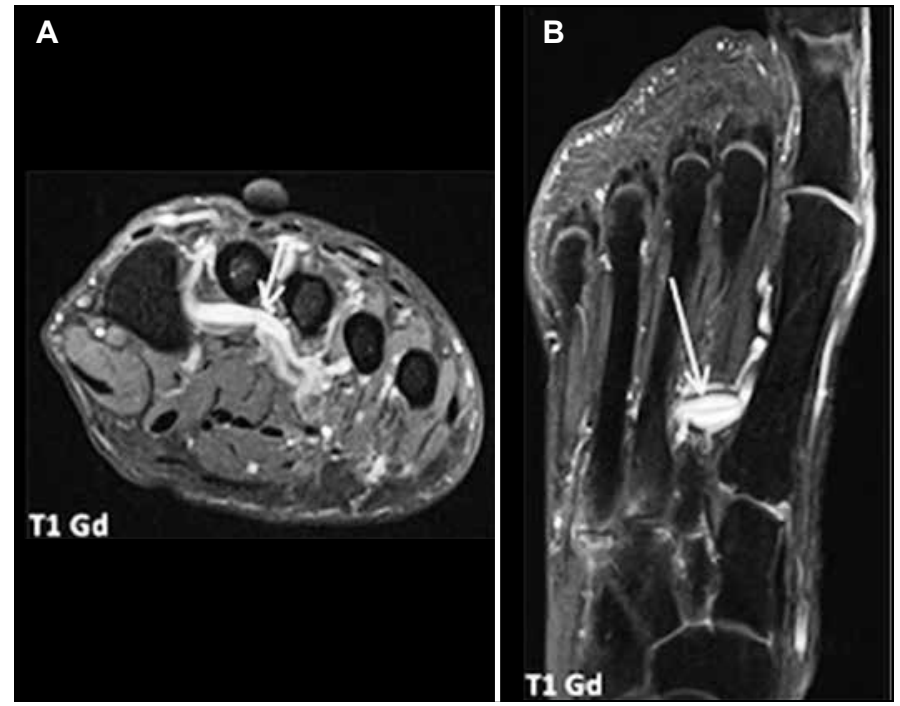

Figure 3 - Male patient, 31 years. Coronal and axial T1W with fat saturation and contrast $(A-B)$ - Collateral veins in the topography of the plantar metatarsal system, in a patient who was diagnosed with deep plantar thrombophlebitis, probably representing a chronic case.

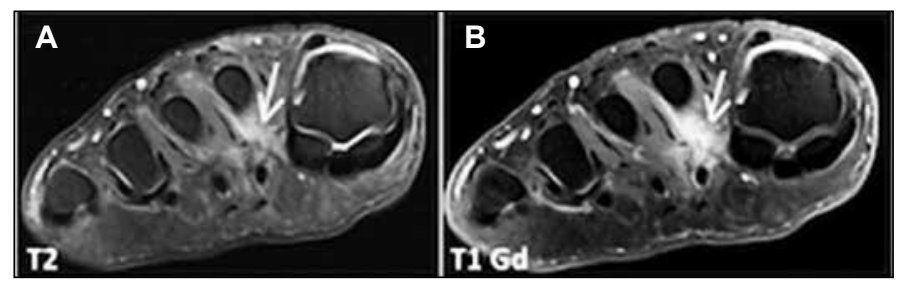

Figure 4 - Male patient, 49 years. Coronal T2WI FSE with fat saturation and T1WI FSE with fat saturation and contrast (A-B) - Perivascular enhancement after gadolinium injection, around the plantar metatarsal vein of the second ray of the right forefoot.
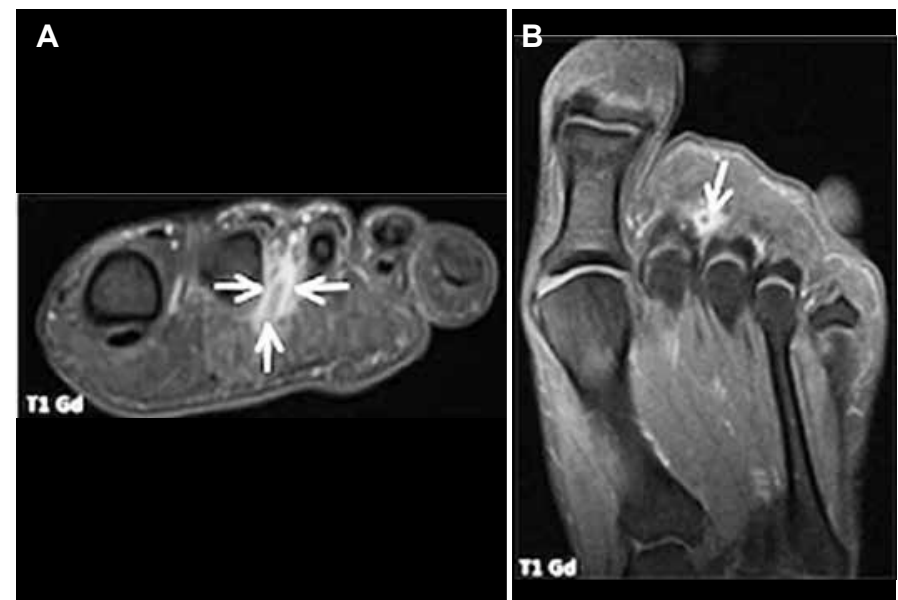

Figure 5 - Female patient, 43 years. Coronal and axial T1WI FSE with fat saturation and contrast (A-B) - Intraluminal filling defect in the plantar digital vein of the second ray of the left forefoot.

Table 2 - Site of thrombophlebitis.

\begin{tabular}{c|l|l}
\hline Affected vein & $\mathbf{n}$ & $\%$ \\
\hline Medial plantar veins & 2 & 10 \\
\hline Lateral plantar veins & 6 & 30 \\
\hline Plantar metatarsal veins & 8 & 40 \\
\hline Plantar digital veins & 4 & 20 \\
\hline
\end{tabular}




\section{DISCUSSION}

The deep plantar venous plexus is composed of multiple veins ${ }^{(3)}$ which are located in the deep layers of the foot, underneath the plantar arch and, therefore, are vulnerable to repetitive trauma during deambulation and in physical activity ${ }^{(4)}$. The plantar digital veins originate from the plexus on the plantar surface of the digits, joining to form the metatarsal veins, located in the metatarsal spaces, which then form the deep plantar venous arch. They follow the plantar arterial arch and give origin to the medial and lateral veins, which, after emitting the great and small saphenous vein, unite behind the medial malleolus to give origin to the posterior tibial veins ${ }^{(3)}$ (Figure 6). The plantar venous plexus is filled quickly when the foot is in a hanging position and empties immediately when a load is supported by the plantar arch. The drainage of blood from the plexus is independent of muscle contraction ${ }^{(4)}$.

The hyperintense signal of the perivascular planes on T2WI probably represents edema or an inflam-

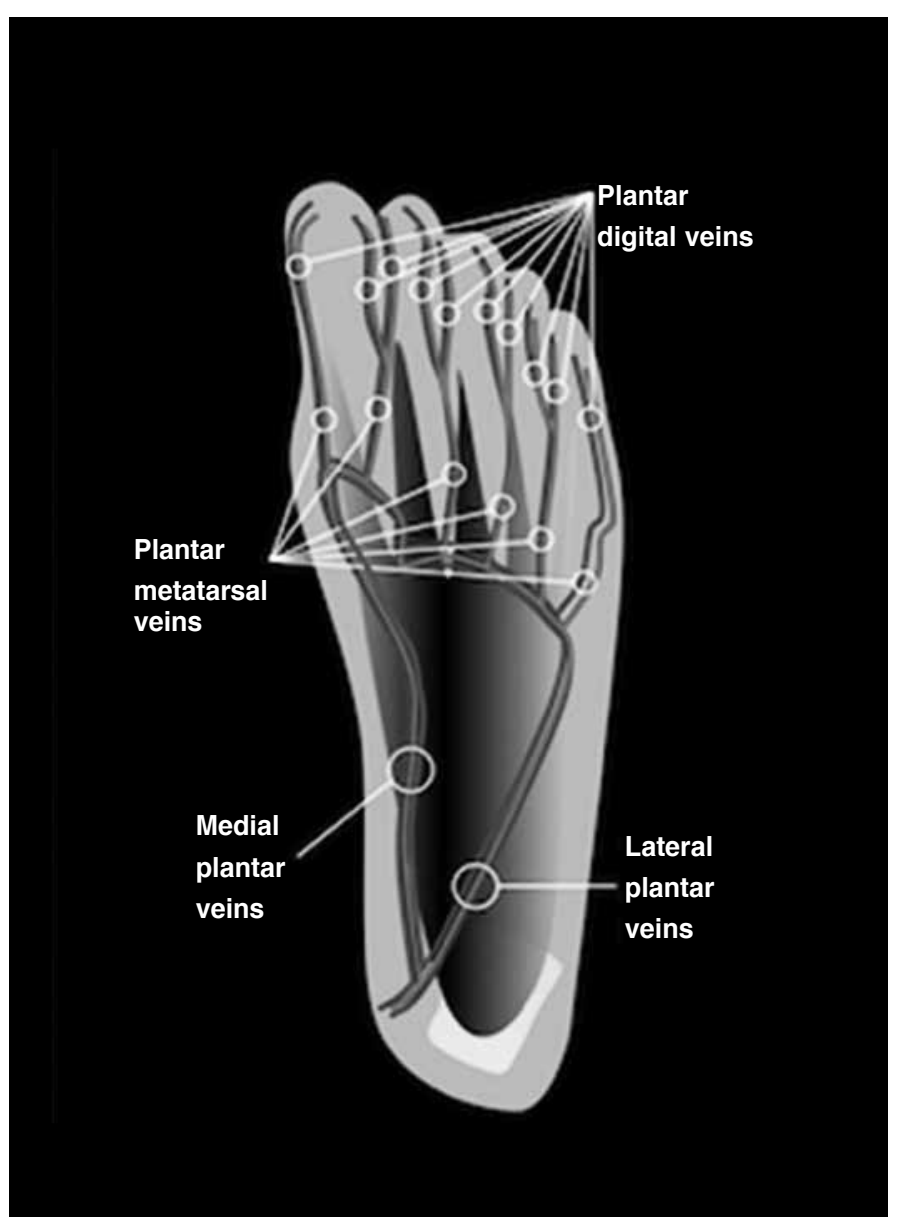

Figure 6 - Simplified diagram of the venous anatomy of the plantar region. matory reaction. Another hypothesis for this hyperintense signal could be related to neural and/or metabolic changes caused by the circulatory deficiency produced by thrombophlebitis.

Plantar thrombophlebitis is an entity with few cases reported in the literature. During our analysis, we found a few isolated case reports ${ }^{(5-7)}$ and others with a larger number of cases described by Bernathova et $\mathrm{al}^{(2)}$ and Barros and Labropoulos ${ }^{(8)}$. Therefore, we believe that this article presents the largest series reported in the English-language literature. It is important to note that the diagnosis in all cases was initially suggested by MRI. Moreover, none of the patients were referred for the exam with clinical suspicion of PT, probably due to the difficulty of diagnosis and because of the lack of knowledge of this entity, even among orthopedists and other medical specialties.

The pathogenesis of this entity is still uncertain, and is related to previous surgery, trauma, paraneoplastic conditions, genetic mutations of the blood coagulation cascade, and excessive physical activity $^{(1)}$. These factors are responsible for changes in one or more of the three components of Virchow's triad, which summarizes the possibilities of a thrombotic event (endothelial damage, for example, trauma; hypercoagulable states, for example, genetic mutations; and blood stagnation, for example, immobilization after surgery $)^{(9,10)}$.

In the series we can see that the segments most frequently involved were the lateral plantar veins, involved in $30 \%(6 / 20)$ of cases and the plantar metatarsal veins, involved in $40 \%$ (8/20), favoring a probable traumatic or compressive origin for thrombophlebitis. Moreover, some of the patients practiced sports activities such as running, which contributes to the possibility of repetitive trauma in the plantar region as a causal factor.

Patients complain of uncharacteristic pain in the plantar region and, for this reason, the clinical diagnosis is difficult, with multiple differentials, such as intermetatarsal bursitis, Morton's neuroma, sesamoiditis, plantar fasciitis, tendon pathologies, ganglion cysts, and stress fracture.

MRI may be very useful in the diagnosis of plantar thrombophlebitis, when clinical suspicion includes any of the differentials for pain in the plantar region. Whereas the clinical picture of PT overlaps with many 
painful conditions of the foot, the main advantage of MRI is its ability to diagnose this condition and exclude all other differentials. Still, in doubtful cases, the use of intravenous contrast may help in the diagnosis.

The main findings of the MRI were perivascular edema (hyperintense signal of the perivenular tissue in T2WI with fat suppression) and, in cases where intravenous contrast was administered, an intraluminal filling defect and enhancement of perivenular tissue (Figure 7).

Although to our knowledge this study has the largest number of cases reported in the Englishlanguage literature, a greater number of cases would be useful to validate the imaging findings of venous thrombophlebitis and could perhaps show other diagnostic signs. Other limitations of our study include the fact that not all cases were assessed by ultrasonography and that the cases were not evaluated after treatment and resolution of symptoms. However, we emphasize that all patients had clinical improvement after treatment for PT.

\section{CONCLUSION}

In magnetic resonance imaging of painful pathologies of the plantar region, it is important to remember the diagnosis of plantar thrombophlebitis, especially
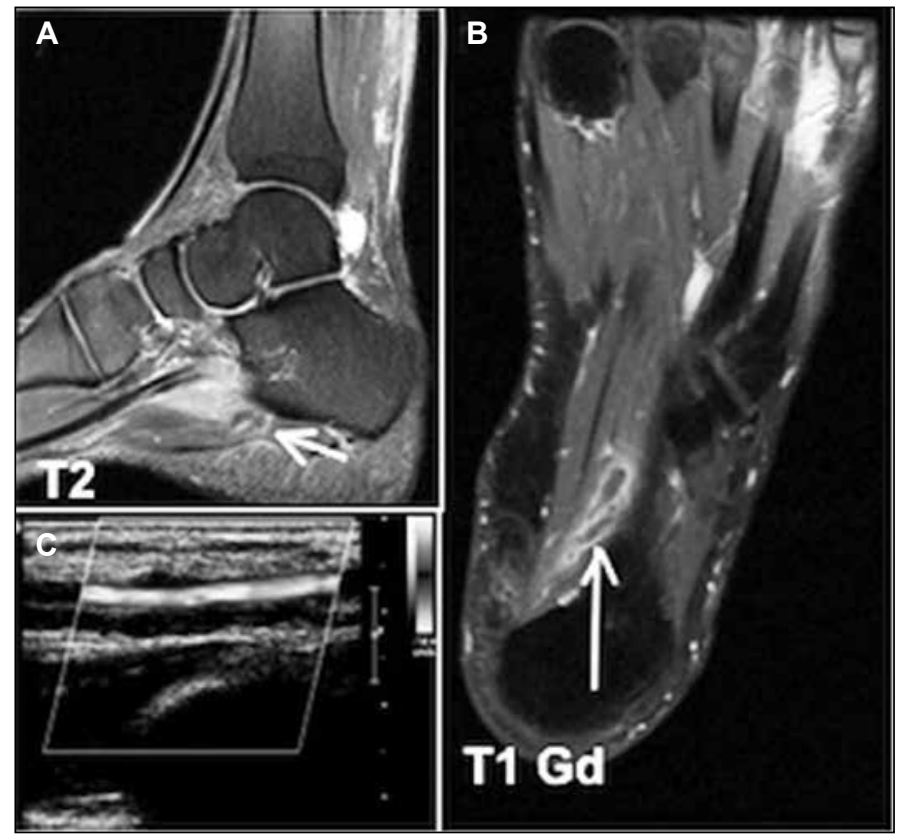

Figure 7 - Male patient, 50 years old, with pain in the plantar region. (A-B) Plantar thrombophlebitis in the topography of the lateral plantar veins with perivascular edema and enhancement, muscle edema, venular ectasia, and intraluminal filling defect. (C) Ultrasound of the lateral plantar region of the foot with color doppler confirming the absence of flow in the lateral plantar vein.

when there is edema along the plantar veins and no other findings suggestive of other diseases. We believe that this may become increasingly recognized with knowledge of the pathology and experience with its signs in imaging (especially MRI).

\section{REFERENCES}

1. Siegal DS, Wu JS, Brennan DD, Challies T, Hochman MG. Plantar vein thrombosis: a rare cause of plantar foot pain. Skeletal Radiol. 2008;37(3):267-9.

2. Bernathova M, Bein E, Bendix N, Bodner G. Sonographic diagnosis of plantar vein thrombosis: report of 3 cases. J Ultrasound Med. 2005;24(1):101-3.

3. Gray H. Anatomy of the human body. Philadelphia: Lea \& Febiger, 1918; Bartleby.com, 2000. Disponivel: www.bartleby.com/107/.

4. White JV, Katz ML, Cisek P, Kreithen J. Venous outflow of the leg: anatomy and physiologic mechanism of the plantar venous plexus. J Vasc Surg. 1996;24(5):819-24.

5. Cavezzi A. Isolated thrombosis of plantar veins. Case report. Minerva Cardioangiol. 1999;47(9):309-13.
6. Legrand MS, Papon X, Leftheriotis G, Saumet JL. [Isolated plantar venous thrombosis. Report of a case]. J Mal Vasc. 1997;22(5):364-5.

7. Long A, Bura-Riviere A, Sapoval M. [Plantar venous thrombosis and anticardiolipin antibody syndrome. Case report]. J Mal Vasc. 2004;29(1):39-40.

8. Barros MV, Labropoulos N. Plantar vein thrombosis--evaluation by ultrasound and clinical outcome. Angiology. 2010;61(1):82-5.

9. Bagot CN, Arya R. Virchow and his triad: a question of attribution. Br J Haematol. 2008;143(2):180-90.

10. Furie B, Furie BC. Mechanisms of thrombus formation. $\mathrm{N}$ Engl J Med. 2008;359(9):938-49. 